\title{
COVID-19 and drug-induced liver injury: a problem of plenty or a petty point?
}

\author{
Joost Boeckmans ${ }^{1} \cdot$ Robim M. Rodrigues $^{1} \cdot$ Thomas Demuyser $^{2} \cdot$ Denis Piérard $^{2} \cdot$ Tamara Vanhaecke $^{1} \cdot$ Vera Rogiers $^{1}$
}

Received: 31 March 2020 / Accepted: 1 April 2020 / Published online: 8 April 2020

c) Springer-Verlag GmbH Germany, part of Springer Nature 2020

In December 2019, a novel coronavirus, the severe acute respiratory syndrome coronavirus 2 (SARS-CoV-2), broke out in the Chinese province of Wuhan and rapidly infected hundreds of thousands of people worldwide. Infected patients mostly exhibit general signs of pneumonia (fever, dyspnea, coughing and desaturation) and a presumably significant number of infected patients are asymptomatic. Yet, about $5-10 \%$ of patients develop critical oxygen deprivation, needing intensive care and intubation with mechanical ventilation (Huang et al. 2020; Raoult et al. 2020). Typical acute phase laboratory findings are present in admitted COVID-19 patients, e.g. elevated c-reactive protein and ferritin. Also, lactate dehydrogenase, aspartate aminotransferase (AST) and alanine aminotransferase (ALT) are often elevated in COVID-19 patients, indicating liver damage (Zhou et al. 2020). Recent reports point to the fact that liver injury, by means of increased aminotransferase levels, more frequently occurred in severe COVID-19 cases compared to patients with mild symptoms (Zhang et al. 2020; Guan et al. 2020; Liu et al. 2020a, b; Chen et al. 2020).

SARS-CoV-2 enters the cell by binding onto angiotensinconverting enzyme 2 (ACE2) (Gurwitz 2020). Until today, no strong evidence is present that ACE2 is expressed in the liver, although a recent, non-peer reviewed, article reported its expression in cholangiocytes (Hamming et al. 2004; Chai et al. 2020).

Retrospective analysis of the earlier-reported SARS-CoV, causing the 'severe acute respiratory syndrome (SARS)' and also infecting humans via ACE2, showed that the disease

Vera Rogiers

vera.rogiers@vub.be

1 Department of In Vitro Toxicology and Dermato-Cosmetology (IVTD), Faculty of Medicine and Pharmacy, Vrije Universiteit Brussel, Laarbeeklaan 103, 1090 Brussels, Belgium

2 Department of Microbiology, Universitair Ziekenhuis Brussel, Laarbeeklaan 101, 1090 Brussels, Belgium was accompanied by abnormal liver function which could be due to viral replication in the liver (Cui et al. 2004). It is indeed known that multiple (respiratory) viruses can disrupt liver functions via $(\mathrm{CD} 8+)$ immune cell-mediated reactions (Adams and Hubscher 2006).

It has been reported that patients suffering from certain viral infections, such as infections caused by hepatitis $\mathrm{C}$ and human immunodeficiency viruses, are more prone for developing drug-induced liver injury (DILI), especially when associated with highly active anti-retroviral therapy (Naidoo et al. 2019; Bonacini 2004). Recently it was postulated that liver impairment in COVID-19 patients could also be drug-related (Zhang et al. 2020). This may have consequences for patients who are treated with novel, potentially hepatotoxic, antiviral drugs and perhaps also antibiotics for bacterial superinfections (Xu et al. 2020a, b). Hydroxychloroquine is applied as an antiviral drug, with sparse evidence in small clinical settings. Yet, viral replication and disease progression would be reduced and overall survival would increase. The effect of hydroxychloroquine on hepatic tissue seems ambiguous, since it is used to treat liver infection with protozoa (i.e. malaria) but cases of fulminant hepatic failure have been reported (Makin et al. 1994; Liu et al. 2020a). Azithromycin would have additional effects to hydroxychloroquine. However, there is little evidence and the underlying mechanisms are unclear (Gautret et al. 2020). Furthermore, azithromycin-induced hepatotoxicity only appears 1-3 weeks after azithromycin initiation (Martinez et al. 2015).

Patients suffering either from diabetes type 1 or 2 , or developing hypertension, of ten receive blood pressure reducing ACE inhibitors and angiotensin II type I receptor blockers, which may lead to ACE2 overexpression. Therefore, although clinical evidence is lacking, it has to be kept in mind that ACE2 upregulation might be at the origin of a higher susceptibility for developing COVID-19 (Fang et al. 2020; Fitzgerald 2020). Furthermore, these patients often suffer from the metabolic syndrome, which is a major 
risk factor for non-alcoholic fatty liver disease (NAFLD). NAFLD can sensitize the liver for hepatotoxicants such as acetaminophen (Michaut et al. 2014; Massart et al. 2017), which is the advised and widely used anti-pyretic symptomatic drug treatment. Although it is actually not known whether a link exists between COVID-19 and liver steatosis, it is worrying that in a recent post-mortem histopathological analysis of a liver biopsy of a COVID19 patient microvesicular liver steatosis was observed (Xu et al. 2020a, b). Even though this finding needs to be confirmed in larger studies, it has also been demonstrated that patients suffering from COVID-19 exhibited increased serum levels of monocyte chemoattractant protein-1 (MCP-1) (Huang et al. 2020), which is a chemokine known to exacerbate steatohepatitis (Gao and Tsukamoto 2016). Consequently, pre-disposing factors for developing steatohepatitis, such as intake of steatosis-inducing drugs (e.g. sodium valproate, amiodarone, tamoxifen and methotrexate) (Boeckmans et al. 2018), could play a role in combined COVID-19 and DILI. Knowing that liver dysfunction and NAFLD are more common in the elderly, who are also more prone to develop severe COVID-19, it is probably wise to carefully monitor COVID-19 patients for drug treatment that could lead to DILI.

In vitro methodologies are well-positioned to study potential DILI of compounds currently being developed against COVID-19. Relatively simple human-relevant cell models can be used to evaluate hepatotoxic properties of (novel) drugs in a relatively short time. This has been robustly demonstrated for a large series of compounds (Rodrigues et al. 2016; Kuna et al. 2018). Also co-morbidities such as NAFLD that increase the risk for developing DILI can be modeled using in vitro methodology (Massart et al. 2017; Boeckmans et al. 2019). Recently, Huh7 and Vero E6 cells in culture have been used to assess the pharmacological effects of remdesivir, chloroquine and hydroxychloroquine, all potential anti-COVID-19 treatments under study (Liu et al. 2020a; Wang et al. 2020). Hence, in vitro methodologies can be easily explored for increasing our knowledge with respect to the pharmacological concern and concomitant toxicological aspects of COVID-19 treatment.

Overall, despite the indications that SARS-CoV-2 infection might have an influence on normal hepatic functions and the fact that drugs are used for the treatment of COVID-19 that have proven hepatotoxic properties (whether or not due to overdosing), more mechanistic studies regarding SARS-CoV-2 entry and replication in liver cells and the potential consequences of medication for vital organs, including the liver, are warranted.

\section{Compliance with ethical standards}

Conflict of interest The authors declare that they have no conflict of interest.

\section{References}

Adams DH, Hubscher SG (2006) Systemic viral infections and collateral damage in the liver. Am J Pathol 168:1057-1059. https:// doi.org/10.2353/ajpath.2006.051296

Boeckmans J, Natale A, Buyl K et al (2018) Human-based systems: mechanistic NASH modelling just around the corner? Pharmacol Res 134:257-267. https://doi.org/10.1016/j.phrs.2018.06.029

Boeckmans J, Buyl K, Natale A et al (2019) Elafibranor restricts lipogenic and inflammatory responses in a human skin stem cellderived model of NASH. Pharmacol Res 144:377-389. https:// doi.org/10.1016/j.phrs.2019.04.016

Bonacini M (2004) Liver injury during highly active antiretroviral therapy: the effect of hepatitis C coinfection. Clin Infect Dis 38:104-108. https://doi.org/10.1086/381453

Chai X, Hu L, Zhang Y et al (2020) Specific ACE2 expression in cholangiocytes may cause liver damage after 2019-NCoV infection. bioRxiv. https://doi.org/10.1101/2020.02.03.931766. (Online pre-print)

Chen G, Wu D, Guo W et al (2020) Clinical and immunologic features in severe and moderate coronavirus disease 2019. J Clin Invest. https://doi.org/10.1172/JCI137244. (In press)

Cui H, Tong X, Li P et al (2004) Serum hepatic enzyme manifestations in patients with severe acute respiratory syndrome: retrospective analysis. World J Gastroenterol 10:1652-1655. https:// doi.org/10.3748/wjg.v10.i11.1652

Fang L, Karakiulakis G, Roth M (2020) Are patients with hypertension and diabetes mellitus at increased risk for COVID-19 infection? Lancet Respir Med. https://doi.org/10.1016/S2213 -2600(20)30116-8. (In press)

Fitzgerald GA (2020) Misguided drug advice for COVID-19. Science 367:1434. https://doi.org/10.1126/science.abb8034

Gao B, Tsukamoto H (2016) Inflammation in alcoholic and nonalcoholic fatty liver disease: friend or foe? Gastroenterology 150:1704-1709. https://doi.org/10.1053/j.gastro.2016.01.025

Gautret P, Lagier J, Parola P et al (2020) Hydroxychloroquine and azithromycin as a treatment of COVID-19: results of an openlabel non-randomized clinical trial. Int J Antimicrob Agents. https ://doi.org/10.1016/j.ijantimicag.2020.105949. (In press)

Guan W, Ni Z, Hu Y et al (2020) Clinical characteristics of coronavirus disease 2019 in China. N Engl J Med. https://doi.org/10.1056/ NEJMoa2002032. (In press)

Gurwitz D (2020) Angiotensin receptor blockers as tentative SARSCoV-2 therapeutics. Drug Dev Res. https://doi.org/10.1002/ ddr.21656. (In press)

Hamming I, Timens W, Bulthuis ML et al (2004) Tissue distribution of ACE2 protein, the functional receptor for SARS coronavirus. a first step in understanding SARS pathogenesis. J Pathol 203:631637. https://doi.org/10.1002/path.1570

Huang C, Wang Y, Li X et al (2020) Clinical features of patients infected with 2019 novel coronavirus in Wuhan, China. Lancet 395:497-506. https://doi.org/10.1016/S0140-6736(20)30183-5

Kuna L, Bozic I, Kizivat T et al (2018) Models of drug induced liver injury (DILI) - current issues and future perspectives. Curr Drug Metab 19:830-838. https://doi.org/10.2174/138920021966618 0523095355

Liu J, Cao R, Xu M et al (2020a) Hydroxychloroquine, a less toxic derivative of chloroquine, is effective in inhibiting SARS-CoV-2 
infection in vitro. Cell Discov 6:16. https://doi.org/10.1038/s4142 1-020-0156-0

Liu W, Tao ZW, Lei W et al (2020b) Analysis of factors associated with disease outcomes in hospitalized patients with 2019 novel coronavirus disease. Chin Med J (Engl). https://doi.org/10.1097/ CM9.0000000000000775. (In press)

Makin AJ, Wendon J, Fitt S et al (1994) Fulminant hepatic failure secondary to hydroxychloroquine. Gut 35:569-570. https://doi. org/10.1136/gut.35.4.569

Martinez MA, Vuppalanchi R, Fontana RJ et al (2015) Clinical and histologic features of azithromycin-induced liver injury. Clin Gastroenterol Hepatol 13:369-376. https://doi.org/10.1016/j. cgh.2014.07.054

Massart J, Begriche K, Moreau C et al (2017) Role of nonalcoholic fatty liver disease as risk factor for drug-induced hepatotoxicity. J Clin Transl Res. 3:212-232. https://doi.org/10.18053/jctre s.03.2017S1.006

Michaut A, Moreau C, Robin MA et al (2014) Acetaminophen-induced liver injury in obesity and nonalcoholic fatty liver disease. Liver Int 34:171-179. https://doi.org/10.1111/liv.12514

Naidoo K, Hassan-Moosa R, Mlotshwa P et al (2019) High rates of drug-induced liver injury in people living with HIV coinfected with tuberculosis (TB) irrespective of antiretroviral therapy timing during antituberculosis treatment: results from the starting antiretroviral therapy at three points in TB tri. Clin Infect Dis. https://doi.org/10.1093/cid/ciz732. (In press)

Raoult D, Zumla A, Locatelli F et al (2020) Coronavirus infections: epidemiological, clinical and immunological features and hypotheses. Cell Stress 4:66-75. https://doi.org/10.15698/cst2020.04.216
Rodrigues RM, Heymans A, De Boe V et al (2016) Toxicogenomicsbased prediction of acetaminophen-induced liver injury using human hepatic cell systems. Toxicol Lett 240:50-59. https://doi. org/10.1016/j.toxlet.2015.10.014

Wang M, Cao R, Zhang L et al (2020) Remdesivir and chloroquine effectively inhibit the recently emerged novel coronavirus (2019$\mathrm{NCoV}$ ) in vitro. Cell Res 30:269-271. https://doi.org/10.1038/ s41422-020-0282-0

$\mathrm{Xu} \mathrm{L}$, Liu J, Lu M et al (2020a) Liver injury during highly pathogenic human coronavirus infections. Liver Int. https://doi.org/10.1111/ liv.14435. (In press)

Xu Z, Shi L, Wang Y et al (2020b) Pathological findings of COVID-19 associated with acute respiratory distress syndrome. Lancet Respir Med. https://doi.org/10.1016/S2213-2600(20)30076-X. (In press)

Zhang C, Shi L, Wang F (2020) Liver injury in COVID-19: management and challenges. Lancet Gastroenterol Hepatol. https://doi. org/10.1016/S2468-1253(20)30057-1. (In press)

Zhou F, Yu T, Du R et al (2020) Clinical course and risk factors for mortality of adult inpatients with COVID-19 in Wuhan, China: a retrospective cohort study. Lancet. https://doi.org/10.1016/S0140 $-6736(20) 30566-3$. (In press)

Publisher's Note Springer Nature remains neutral with regard to jurisdictional claims in published maps and institutional affiliations. 